\title{
Adrenergic-mediated increases in INHBA drive CAF phenotype and collagens
}

\author{
Archana S. Nagaraja, ${ }^{1}$ Robert L. Dood, ${ }^{1}$ Guillermo Armaiz-Pena, ${ }^{1}$ Yu Kang, ${ }^{1}$ Sherry Y. Wu, ${ }^{1}$ \\ Julie K. Allen, ${ }^{1}$ Nicholas B. Jennings, ${ }^{1}$ Lingegowda S. Mangala, ${ }^{1}$ Sunila Pradeep, ${ }^{1}$ Yasmin Lyons, ${ }^{1}$ \\ Monika Haemmerle, ${ }^{1}$ Kshipra M. Gharpure, ${ }^{1}$ Nouara C. Sadaoui,, ${ }^{1}$ Cristian Rodriguez-Aguayo, ${ }^{2,3}$ \\ Cristina Ivan, ${ }^{2}$ Ying Wang, ${ }^{4}$ Keith Baggerly, ${ }^{4}$ Prahlad Ram, ${ }^{5}$ Gabriel Lopez-Berestein, ${ }^{2,3}$ Jinsong Liu, ${ }^{6}$ \\ Samuel C. Mok, ${ }^{1}$ Lorenzo Cohen, ${ }^{7}$ Susan K. Lutgendorf, ${ }^{8}$ Steve W. Cole, ${ }^{9}$ and Anil K. Sood ${ }^{1,2,10}$ \\ 'Department of Gynecologic Oncology and Reproductive Medicine, ${ }^{2}$ Center for RNAi and Non-Coding RNA, ${ }^{3}$ Department of \\ Experimental Therapeutics, ${ }^{4}$ Department of Bioinformatics and Computational Biology, ${ }^{5}$ Department of Systems Biology, \\ ${ }^{6}$ Department of Pathology, and 'Department of Palliative, Rehabilitation, and Integrative Medicine, The University of \\ Texas MD Anderson Cancer Center, Houston, Texas, USA. ${ }^{8}$ Departments of Psychological and Brain Sciences, Obstetrics \\ and Gynecology, and Holden Comprehensive Cancer Center, University of lowa, lowa City, lowa, USA. ${ }^{9}$ Department of \\ Medicine and Jonsson Comprehensive Cancer Center, University of California, Los Angeles School of Medicine, UCLA \\ Molecular Biology Institute, and Norman Cousins Center, Los Angeles, California, USA. ${ }^{10}$ Department of Cancer Biology, \\ The University of Texas MD Anderson Cancer Center, Houston, Texas, USA.
}

Adrenergic signaling is known to promote tumor growth and metastasis, but the effects on tumor stroma are not well understood. An unbiased bioinformatics approach analyzing tumor samples from patients with known biobehavioral profiles identified a prominent stromal signature associated with cancer-associated fibroblasts (CAFs) in those with a high biobehavioral risk profile (high Center for Epidemiologic Studies Depression Scale [CES-D] score and low social support). In several models of epithelial ovarian cancer, daily restraint stress resulted in significantly increased CAF activation and was abrogated by a nonspecific $\beta$-blocker. Adrenergic signaling-induced CAFs had significantly higher levels of collagen and extracellular matrix components than control tumors. Using a systems-based approach, we found INHBA production by cancer cells to induce CAFs. Ablating inhibin $\beta$ A decreased CAF phenotype both in vitro and in vivo. In preclinical models of breast and colon cancers, there were increased CAFs and collagens following daily restraint stress. In an independent data set of renal cell carcinoma patients, there was an association between high depression (CES-D) scores and elevated expression of ACTA2, collagens, and inhibin $\beta$ A. Collectively, our findings implicate adrenergic influences on tumor stroma as important drivers of CAFs and establish inhibin $\beta$ A as an important regulator of the CAF phenotype in ovarian cancer.

Conflict of interest: The authors have declared that no conflict of interest exists.

Submitted: January 26, 2017 Accepted: July 6, 2017 Published: August 17, 2017

\section{Reference information:} JCI Insight. 2017;2(16):e93076 https://doi.org/10.1172/jici. insight.93076.

\section{Introduction}

Mounting clinical and preclinical evidence has shown that psychosocial factors can trigger chronic adrenergic signaling within tumors and promote tumor growth and metastasis in many tumor types (1-5). Activation of the sympathetic nervous system leads to release of stress hormones such as epinephrine and norepinephrine (NE), which signal via adrenergic receptors on tumor cells, resulting in diminished efficacy of conventional chemotherapy and promotion of tumor metastasis, inflammation, and other prosurvival pathways (6-12). Although molecular events that have direct effects on tumor cells have been well documented, the effects of adrenergic signaling on the tumor stroma are not well understood. The goal of this study was to identify the effects of chronic adrenergic signaling on cancer-associated fibroblasts (CAFs) in the tumor microenvironment.

CAFs can contribute substantially to tumor growth. By depositing extracellular matrix (ECM) components, fibroblasts can potentiate migration and invasion of cancer cells and decrease access of chemotherapy and immunotherapy drugs as well as cytotoxic T cells (13-17). The ability of cancer cells to educate normal fibroblasts toward a CAF phenotype and transform resident fibroblasts to a reactive stromal phenotype is well known, but the unique role of chronic stress in mediating this effect is not understood $(13,16)$. 
A

Inflammatory response

Collagen

ycosaminoglycan binding

Growth Factor binding

Extracellular structure organization

Epidermis development

Protein complex binding

Polysaccharide binding

Hemostasis

Blood coagulation

Cell substrate adhesion

Response to other organisms

Extracellular matrix part

Vascular development

Blood vessel development

Proteinaceous extracellular matrix

Extracellular matrix

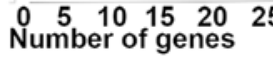

Neurogenesis $\square$ Angiogenesis

Immune Response $\square$ ECM-related pathways

Cell-cell communications

C

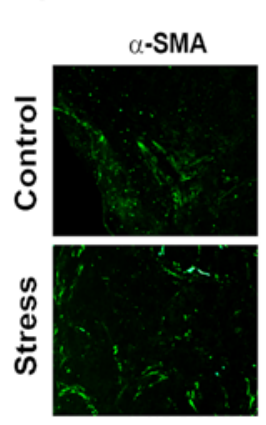

\section{Skov3-ip1} CD31

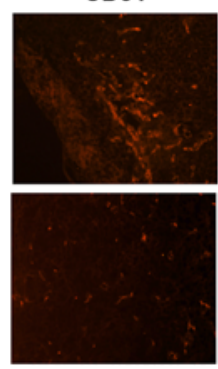

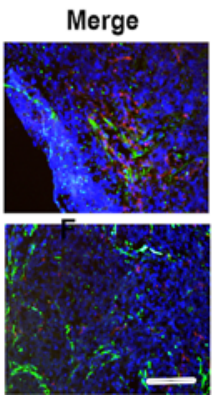

D

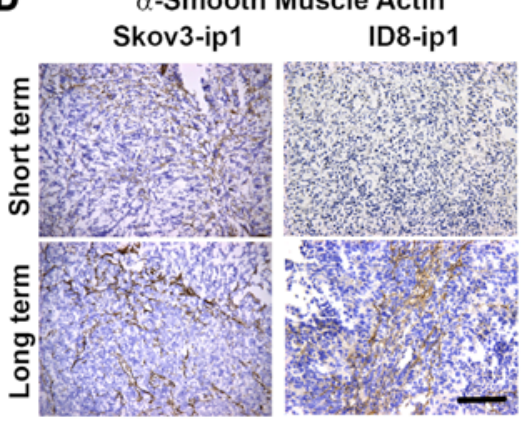

Figure 1. Chronic stress accelerates induction of the cancer-associated fibroblast (CAF) phenotype in ovarian carcinoma. (A) Results from NetWalker analysis to identify networks of upregulated genes in ovarian cancer patients with high Center for Epidemiologic Studies Depression Scale [CES-D] (i.e., depression) scores and low social support. (B) Expression of $\alpha$-smooth muscle actin ( $\alpha$-SMA, a CAF marker) in micrographs of representative tumors from control and restraint-stressed mice in adrenergic receptor-positive Skov3-ip1, HeyA8, and ID8-ip1 models. (C) Expression of $\alpha$-SMA and CD31 (blood vessel marker) in micrographs of representative Skov3-ip1 tumors from control and stressed mice. (D) Expression of $\alpha$-SMA in micrographs of representative tumors from mice subjected to short-term (7 days) or long-term (21 days) stress. Scale bars: $100 \mu \mathrm{m} . n=5 /$ group for all data.

In ovarian cancer, crosstalk between CAFs and cancer cells is associated with production of CAF-specific growth factors and ECM that can support tumor growth and metastasis (18).

We identified a prominent stromal signature, indicated by elevated expression of CAF-related genes and several collagens, in tumors from patients with a high biobehavioral risk composite score. Using bioinformatics analyses, we found that induction of the CAF phenotype is due primarily to inhibin $\beta$ A (INHBA) production by cancer cells after NE stimulation. We further identified the critical role that the $\beta_{2}$-adrenergic receptor/cAMP responsive element binding protein/INHBA (ADRB2/CREB/INHBA) axis plays in mediating a stress hormone-induced response in tumor stroma. Silencing inhibin $\beta \mathrm{A}$ in tumor cells in preclinical models reversed increases in levels of collagen within tumors during chronic adrenergic stimulation, leading to significantly decreased tumor burden.

\section{Results}

Stromal signature in ovarian tumors from patients with chronic adrenergic activation. To identify potential pathways affected by adrenergic signaling, we first compared high-grade serous cancers (HGSCs) from 5 patients with high biobehavioral risk (Center for Epidemiologic Studies Depression Scale [CES-D] score $\geq 16$ and SPS attachment $<15$ ) with tumors from 5 patients with low biobehavioral risk (CES-D $<16$ and SPS attachment $\geq 15$ ); these data were collected from a prospective cohort study (GEO GSE9116) (19). Selecting for genes that were significantly upregulated in the tumors of patients with high biobehavioral risk (by at least 2 -fold at the $P<0.05$ level), we then performed a protein network analysis using NetWalker. Strikingly, we uncovered a strong signature indicative of a reactive stroma in tumors from patients with high biobehavioral risk (Figure 1A and Table 1). It should be noted that those patients with high biobehavioral risk also had significantly elevated tumor norepinephrine (NE) (19). Comparing the top 
200 genes from these tumors with gene expression data comparing microdissected CAFs and normal fibroblasts (GEO GSE40643), we found 22 genes that were common to the high biobehavioral risk group and the CAFs, suggesting that CAFs are indeed enriched during chronic adrenergic signaling (Table 2 and Supplemental Figure 1A; supplemental material available online with this article; https://doi.org/10.1172/jci. insight.93076DS1).

To determine the biological relevance of these findings, we used ADRB-positive cell lines in orthotopic mouse models of both human (Skov3-ip1 and HeyA8) and murine (ID8-ip1) epithelial ovarian cancer. We induced chronic stress in the animals by using a well-characterized physical-restraint system $(11,20)$, which is known to result in sustained elevations of NE in tissues (20). In tumors obtained at necropsy, samples from mice exposed to chronic restraint stress had significantly greater numbers of intratumoral cells positive for the CAF marker, $\alpha$-smooth muscle actin ( $\alpha$-SMA), in all models (Skov3-ip1: 4.23-fold increase, $P<0.05$; HeyA8: 1.88-fold increase, $P<0.01$; ID8-ip1: 2.9-fold increase, $P<0.05$ ) (Figure 1B and ref. 14). Since endothelial cells may also stain for $\alpha$-SMA, we also costained for CD31 and $\alpha$-SMA. The significantly higher $\alpha$-SMA-positive signal in tumors from mice that underwent chronic stress compared with controls was independent of CD31-positive cells, indicating that restraint stress induced an increase in the CAF phenotype (CD31-independent $\alpha$-SMA ${ }^{+}$cells: 1.8 -fold increase) (Figure $1 \mathrm{C}$ and refs. 21, 22). We also evaluated the tumors for other CAF markers including fibroblast-activated protein (FAP), desmin, and vimentin and found a similar increase in its expression under restraint stress in the Skov3-ip1 model (Supplemental Figure 1B). The CAF phenotype was more pronounced in mice stressed for 21 days (long-term stress) than in those stressed for 7 days (short-term stress) (Skov3-ip1: 2.1fold increase, $P<0.05$; ID8-ip 1: 2.2-fold increase, $P<0.05$ ) (Figure 1D). CAFs were detected in primary as well as all metastatic sites in these preclinical models of ovarian cancer and the CAF content was increased by restraint stress compared with controls in the Skov3-ip1 model ( 2 -fold increase, $P<0.05$ ) (Supplemental Figure 1C).

Promotion of CAF phenotype by chronic adrenergic signaling. Catecholamines such as $\mathrm{NE}$ can trigger signaling in tumor and other cell types via ADRB receptors (1). To further determine whether the CAF phenotype was due to direct or indirect effects of $\beta$-adrenergic signaling on fibroblasts, we used an ADRB-null tumor model (A2780). There was no significant difference in $\alpha$-SMA expression between tumors from control and restraint-stress groups, indicating the important role of adrenergic signaling in tumor cells for driving the stress-induced CAF phenotype (A2780: 1.1-fold change, $P>0.05$ ) (Figure 2A).

To further assess the distinct roles of epinephrine versus NE signaling as the primary catecholaminergic driver of the CAF phenotype under chronic stress, we assessed the expression of $\alpha$-SMA after chronic restraint stress in tumors from adrenalectomized mice that had been injected with ID8-ip1 cells. In these animals, the adrenal glands were surgically removed, eliminating the primary source of circulating epinephrine, but preserving the primary source of NE (which is released predominately from local peripheral nerves; ref. 23). The adrenalectomized mice had significant increases in $\alpha$-SMA levels under restraint stress (2.5- and 3.1-fold increases over controls in sham surgery and adrenalectomy groups, respectively; $P<0.05$ ) (Figure $2 \mathrm{~B}$ ), implying a primary role of $\mathrm{NE}$. To further define the role of adrenergic receptors, we first treated tumor bearing mice with PBS or propranolol, a nonspecific $\beta$-blocker that blocks all downstream $\beta$-adrenergic signaling in cells. Propranolol abrogated the stress-mediated increases in $\alpha$-SMA level in the HeyA8 model (PBS: 2.2-fold increase; $P<0.05$, propranolol: 0.87 -fold change, $P>0.05$ ) and reduced gene expression of CAF markers in the Skov3-ip1 model (Figure 2C). Conversely, treatment of tumor-bearing mice with either isoproterenol (nonspecific $\beta$ agonist) or terbutaline (ADRB2-specific agonist) increased CAFs by 2.5- and 3.1-fold, respectively, in tumors (Figure 2D). To further determine which receptor is responsible for the CAF phenotype, we specifically targeted ADRB2 since this is the primary receptor on tumor cells through which NE exerts its pro-tumoral effects $(20,24,25)$. In the HeyA8 mouse model in which ADRB2 was silenced in tumor cells by siRNA incorporated into nanoliposomes, stress-mediated increases in $\alpha$-SMA expression were abrogated (Figure $2 \mathrm{E}$ ).

Next, we examined the consequences of tumor cell adrenergic stimulation for fibroblast activation in vitro. We used NOF151, a normal fibroblast cell line derived from normal ovary that has been previously characterized (26). Similar to fibroblasts in preclinical models, these cells have low expression of adrenergic receptors and therefore do not respond to direct NE treatment, as indicated by lack of increase in cAMP levels (Supplemental Figure 2, A and B). NOF151 also did not show increases in expression of CAF markers $A C T A 2, F A P$, and $S 100 A 4$ after direct treatment with NE (Supplemental Figure 2C). Next, NOF151 cells were exposed to either 
Table 1. Top networks upregulated in tumors from patients with high CES-D scores compared to those with low CES-D scores, with at least 7 genes involved

\begin{tabular}{|c|c|c|c|}
\hline Annotation ID & Functional Annotation & $\begin{array}{l}\text { Number of } \\
\text { occurrences }\end{array}$ & Genes \\
\hline ५0:0031012 & extracellular matrix & 20 & $\begin{array}{l}\text { COL5A3, COL5A1, COL1A2, LGALS1, COL1A1, MMP11, PI3, SPARC, THBS1, COL8A1, COL8A2, CTGF, } \\
\text { TIMP2, TNC, DCN, COL6A1, COL3A1, COL17A1, VWF, CTSD }\end{array}$ \\
\hline GO:0005578 & proteinaceous extracellular matrix & 18 & $\begin{array}{l}\text { COL5A3, COL5A1, COL1A2, LGALS1, COL1A1, MMP11, PI3, SPARC, COL8A1, COL8A2, CTGF, TIMP2, } \\
\text { TNC, DCN, COL6A1, COL3A1, COL17A1, VWF }\end{array}$ \\
\hline G0:0001568 & blood vessel development & 13 & $\begin{array}{c}\text { COL5A1, COL1A2, COL1A1, ITGA5, THBS1, ANPEP, COL8A1, } \\
\text { COL8A2, C5AR1, CTCF, TGFBR2, COL3A1, LYL1 }\end{array}$ \\
\hline GO:0001944 & vasculature development & 13 & $\begin{array}{l}\text { COL5A1, COL1A2, COL1A1, ITGA5, THBS1, ANPEP, COL8A1, } \\
\text { COL8A2, C5AR1, CTGF, TGFBR2, COL3A1, LYL1 }\end{array}$ \\
\hline GO:0044420 & extracellular matrix part & 13 & $\begin{array}{l}\text { COL5A3, COL5A1, COL1A2, COL1A1, SPARC, COL8A1, COL8A2, } \\
\text { TIMP2, TNC, DCN, COL6A1, COL3A1, COL17A1 }\end{array}$ \\
\hline ५0:0051707 & response to other organism & 13 & CCL8, ACTA2, CD14, LBP, PLA2C2A, GPX3, SOD2, CCL5, C5AR1, CXCL12, HCK, DCN, NFKBIA \\
\hline G0:0031589 & cell-substrate adhesion & 13 & $\begin{array}{l}\text { COL5A3, LGALS1, ITGA11, COL1A1, ITCB5, ITGA3, THBS1, ITGB2, } \\
\text { COL8A1, CTGF, COL3A1, COL17A1, VWF }\end{array}$ \\
\hline GO:0007596 & blood coagulation & 13 & $\begin{array}{l}\text { COL1A2, COL1A1, RAC2, ITCA5, ITCA3, SPARC, THBS1, } \\
\text { ITCB2, TFPI, SLC16A3, PECAM1, COL3A1, VWF }\end{array}$ \\
\hline GO:0007599 & hemostasis & 13 & $\begin{array}{l}\text { COL1A2, COL1A1, RAC2, ITCA5, ITGA3, SPARC, THBS1, } \\
\text { ITCB2, TFPI, SLC16A3, PECAM1, COL3A1, VWF }\end{array}$ \\
\hline G0:0030247 & polysaccharide binding & 11 & COL5A3, COL5A1, SUSD2, CCL8, THBS1, CD14, ABP1, CTCF, TGFBR2, NCAN, DCN \\
\hline G0:0032403 & protein complex binding & 11 & COL5A1, ITCB5, ITGA5, THBS1, CTGF, TIMP2, HCLS1, COL3A1, VWF, NFKBIA, CTSB \\
\hline G0:0008544 & epidermis development & 11 & COL5A3, COL5A1, COL1A2, COL1A1, LTB, EVPL, KRT17, PPL, CTGF, COL3A1, COL17A1 \\
\hline G0:0043062 & extracellular structure organization & 10 & COL5A3, COL5A1, COL1A2, COL1A1, MYH11, COL8A2, CTGF, TNC, NCAN, COL3A1 \\
\hline G0:0019838 & growth factor binding & 10 & COL5A1, COL1A2, COL1A1, IGFBP3, THBS1, IGFBP4, CTGF, TGFBR2, COL6A1, COL3A1 \\
\hline G0:0005539 & glycosaminoglycan binding & 10 & COL5A3, COL5A1, CCL8, THBS1, CD14, ABP1, CTGF, TGFBR2, NCAN, DCN \\
\hline GO:0005581 & collagen & 10 & COL5A3, COL5A1, COL1A2, COL1A1, COL8A1, COL8A2, DCN, COL6A1, COL3A1, COL17A1 \\
\hline G0:0006954 & inflammatory response & 10 & ALOX5AP, ALOX5, CCL8, THBS1, IGFBP4, CD14, ITCB2, LBP, PLA2C2A, CCL5 \\
\hline G0:0007409 & axonogenesis & 10 & COL5A1, COL1A2, COL1A1, RAC2, ITCA5, MYH11, UCHL1, NCAN, COL6A1, COL3A1 \\
\hline С0:0009617 & response to bacterium & 9 & CD14, LBP, PLA2G2A, SOD2, CCL5, C5AR1, HCK, DCN, NFKBIA \\
\hline ५0:0061061 & muscle structure development & 9 & LGALS1, ITGA11, IGFBP3, MYH11, LMNA, TNC, DCN, SDC1, CTSB \\
\hline G0:0007160 & cell-matrix adhesion & 9 & COL5A3, ITGA11, ITGB5, ITGA3, THBS1, ITGB2, CTGF, COL3A1, COL17A1 \\
\hline GO:0007411 & axon guidance & 9 & COL5A1, COL1A2, COL1A1, RAC2, ITCA5, MYH11, NCAN, COL6A1, COL3A1 \\
\hline GO:0051259 & protein oligomerization & 9 & COL1A2, ALOX5AP, COL1A1, GPX3, SOD2, CRYAB, CCL5, COL6A1, VWF \\
\hline G0:0010035 & response to inorganic substance & 8 & ALOX5AP, FOSB, COL1A1, THBS1, GPX3, SOD2, HP, SDC1 \\
\hline G0:0001525 & angiogenesis & 8 & ITGA5, THBS1, ANPEP, COL8A1, COL8A2, C5AR1, CTGF, TGFBR2 \\
\hline GO:2000145 & regulation of cell motility & 8 & COL1A1, IGFBP3, LMNA, THBS1, LBP, CCL5, C5AR1, CXCL12 \\
\hline ५०:0071702 & organic substance transport & 8 & THBS1, HK3, LBP, ABCC3, SLC04A1, SLC16A3, SLC5A8, NFKBIA \\
\hline G0:0030198 & extracellular matrix organization & 8 & COL5A3, COL5A1, COL1A2, COL1A1, MYH11, COL8A2, CTGF, COL3A1 \\
\hline G0:0030168 & platelet activation & 8 & COL1A2, COL1A1, RAC2, SPARC, THBS1, PECAM1, COL3A1, VWF \\
\hline GO:0005178 & integrin binding & 8 & COL5A1, ITGB5, ITGA5, THBS1, CTGF, TIMP2, COL3A1, VWF \\
\hline G0:0048514 & blood vessel morphogenesis & 8 & ITGA5, THBS1, ANPEP, COL8A1, COL8A2, C5AR1, CTGF, TGFBR2 \\
\hline GO:0048534 & $\begin{array}{l}\text { hemopoietic or lymphoid organ } \\
\text { development }\end{array}$ & 8 & MAFB, LTB, SOD2, CCL5, TGFBR2, HCLS1, LYL1, NFKBIA \\
\hline G0:0030334 & regulation of cell migration & 8 & COL1A1, IGFBP3, LMNA, THBS1, LBP, CCL5, C5AR1, CXCL12 \\
\hline GO:0051270 & $\begin{array}{c}\text { regulation of cellular component } \\
\text { movement }\end{array}$ & 8 & COL1A1, IGFBP3, LMNA, THBS1, LBP, CCL5, C5AR1, CXCL12 \\
\hline GO:0010324 & membrane invagination & 7 & DNM1, TGM2, THBS1, CD14, LBP, PECAM1, HCK \\
\hline G0:0001501 & skeletal system development & 7 & COL1A2, COL1A1, IGFBP4, CTGF, TGFBR2, PAPSS2, COL3A1 \\
\hline G0:0060548 & negative regulation of cell death & 7 & THBS1, SOD2, CRYAB, CCL5, CXCL12, NFKBIA, CTSB \\
\hline G0:0002237 & response to molecule of bacterial origin & 7 & CD14, LBP, SOD2, CCL5, C5AR1, DCN, NFKBIA \\
\hline ५0:0045321 & leukocyte activation & 7 & TNFSF14, THBS1, HLA-DRB1, LBP, CCL5, TGFBR2, LYL1 \\
\hline G0:0090066 & regulation of anatomical structure size & 7 & ALOX5, IGFBP3, ACTA2, IGFBP4, SOD2, CXCL12, CTCF \\
\hline GO:0018193 & peptidyl-amino acid modification & 7 & NCOA3, ITGA5, ITCB2, CCL5, TGFBR2, DCN, HCLS1 \\
\hline G0:0043235 & receptor complex & 7 & ITGA11, ITGB5, ITGA5, ITGA3, CD14, ITGB2, TGFBR2 \\
\hline G0:0030097 & hemopoiesis & 7 & MAFB, SOD2, CCL5, TGFBR2, HCLS1, LYL1, NFKBIA \\
\hline G0:0048545 & response to steroid hormone stimulus & 7 & COL1A1, THBS1, GPX3, CCL5, CTGF, PTCDS, SDC1 \\
\hline G0:0005126 & cytokine receptor binding & 7 & CCL8, LTB, TNFSF14, ITGA5, CCL5, CXCL12, TGFBR2 \\
\hline GO:0005604 & basement membrane & 7 & COL5A1, SPARC, COL8A1, COL8A2, TIMP2, TNC, COL17A1 \\
\hline G0:0007229 & integrin-mediated signaling pathway & 7 & ITGA11, ITGB5, ITGA5, ITGA3, ITGB2, CTGF, COL3A1 \\
\hline G0:0006897 & endocytosis & 7 & DNM1, TCM2, THBS1, CD14, LBP, PECAM1, HCK \\
\hline
\end{tabular}

CES-D, Center for Epidemiologic Studies Depression Scale. 
A

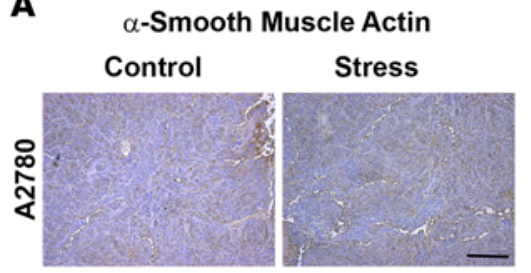

B

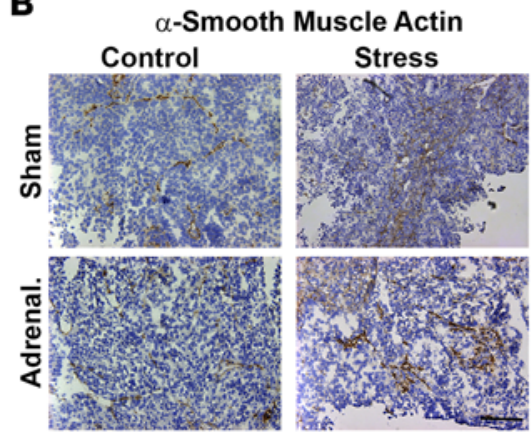

C

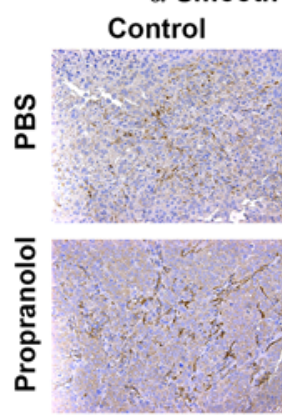
Control

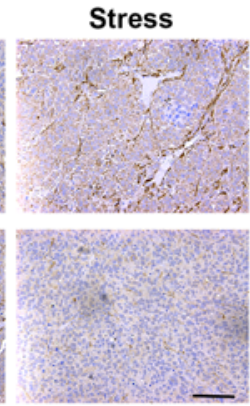

D

\section{a-Smo
Control}

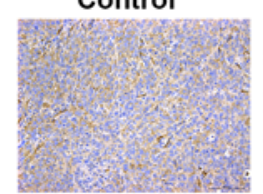

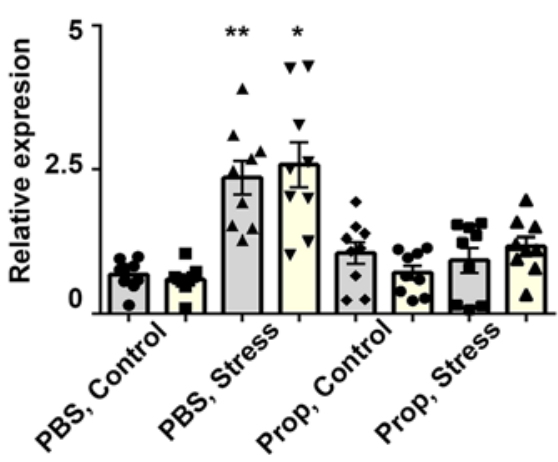

E
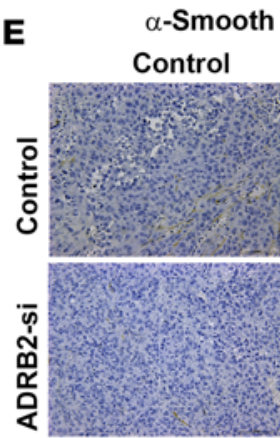

ACTA2

FAP
Figure 2. Induction of the cancerassociated fibroblast (CAF) phenotype in ovarian carcinoma is due to the indirect effects of adrenergic signaling.

(A) Expression of CAF marker $\alpha$-smooth muscle actin ( $\alpha-S M A)$ in micrographs of representative tumors from control and restraint-stressed mice in the $\beta$-adrenergic receptor-negative (ADRBnegative) A2780 (orthotopic) model. (B) Expression of $\alpha$-SMA in micrographs of representative ID8 tumors from control and stressed mice that underwent adrenalectomy (Adrenal.) or sham surgery. (C) Left: Expression of $\alpha$-SMA in micrographs. Right: mRNA expression of ACTA2 and FAP of representative HeyA8 tumors from control and stressed mice treated with the nonspecific $\beta$-blocker propranolol or phosphate-buffered saline solution (PBS, controls). (D) Expression of $\alpha$-SMA in micrographs of representative HeyA8 tumors from control and stressed mice treated with PBS (control), nonspecific $\beta$-agonist isoproterenol, or ADRB2-specific agonist terbutaline. (E) Expression of $\alpha$-SMA in micrographs of representative HeyA8 tumors from control and stressed mice treated with control or ADRB2 siRNA loaded into DOPC nanoliposomes. Scale bars: $100 \mu \mathrm{m} . n=5$ / group for all micrographs, $n=8$ for gene expression data of ACTA2 and FAP (1-way ANOVA for statistical significance). ${ }^{*} P<$ $0.05,{ }^{* *} P<0.01$

control medium conditioned by untreated cancer cells or medium conditioned by NE-treated cancer cells. There was a substantial increase in expression of CAF markers ACTA2, FAP, and S100A4 RNA and $\alpha$-SMA protein (Supplemental Figure 2, D and E; see complete unedited blots in the supplemental material). Two characteristics of transformed fibroblasts in vitro are increased production of proinflammatory cytokines and increased migration $(13,27)$. Conditioned medium from NE-treated cancer cells increased migratory potential (Supplemental Figure $2 \mathrm{~F}$ ) and expression of proinflammatory cytokines such as MCP1, CXCL1, MIF, GM-CSF, and G-CSF (Supplemental Figure 2G) in NOF151 cells. Taken together, these results demonstrate that NE exposure induces tumor cells to express soluble factors that enhance activation of normal fibroblasts. Consistent with the adrenergic receptor specificity documented above in vivo, NOF151 cells exposed in vitro to medium from isoproterenolor terbutaline-treated cancer cells showed greater $\alpha$-SMA induction than those exposed to medium conditioned by nontreated cells (Supplemental Figure 3A). NOF151 cells exposed to conditioned medium from ADRB2silenced cancer cells did not show increases in CAF marker ACTA2 (Supplemental Figure 3B).

Stromal effects of chronic stress. To study the biological consequences of fibroblast activation by chronic stress, we next used NetWalker-unbiased gene-interaction analysis to identify upregulated genes in a patient expression array from GSE9116 (Figure 3A). From this analysis and the results shown in Table 2, we identified an increased collagen signature in tumors from patients with high biobehavioral risk. Masson trichrome and Sirius staining of Skov3-ip1 and HeyA8 tumors confirmed elevated collagen deposition after restraint stress in the in vivo mouse model (increase of 2.1-fold 
Table 2. Genes common to GSE9116 (ovarian cancer patients with high biobehavioral risk score) and GDS40643 (microdissected cancerassociated fibroblasts [CAFs]), arranged alphabetically

\begin{tabular}{|c|c|c|c|}
\hline Affymetrix & Symbol & Patient Samples & Gene array for CAFs \\
\hline 202207_at & $A R L 4 C$ & 3.05 & 3.03 \\
\hline 212077_at & CALD1 & 2.12 & 2.27 \\
\hline 201852_x_at & COL3A1 & 2.15 & 4.399 \\
\hline 212489_at & COL5A1 & 2.09 & 3.809 \\
\hline 221730_at & COL5A2 & 2.33 & 4.267 \\
\hline 209101_at & CTGF & 4.52 & 2.773 \\
\hline 213274_s_at & CTSB & 2.12 & 2.691 \\
\hline 210764_s_at & CYR61 & 2.34 & 2.305 \\
\hline 219454_at & EGFL6 & 2.71 & 3.673 \\
\hline 211719_x_at & FN1 & 2.28 & 2.777 \\
\hline 216560_x_at & IGLC1 & 2.73 & 3.048 \\
\hline 210511_s_at & INHBA & 2.14 & 3.482 \\
\hline 203417_at & MFAP2 & 2.08 & 2.52 \\
\hline 209596_at & MXRA5 & 2.94 & 4.471 \\
\hline 201058_s_at & MYL9 & 2.21 & 2.151 \\
\hline 202620_s_at & PLOD2 & 2.14 & 2.562 \\
\hline 210809_s_at & POSTN & 2.88 & 4.933 \\
\hline 218723_s_at & RGCC & 2.03 & 2.767 \\
\hline 203889_at & SCG5 & 4.31 & 2.185 \\
\hline 212667_at & SPARC & 2.07 & 2.984 \\
\hline 203083_at & THBS2 & 2.09 & 2.568 \\
\hline
\end{tabular}

for both, $P<0.05$ ) (Figure 3B and Supplemental Figure 4A). Stress-mediated collagen deposition in tumors was largely abrogated by propranolol treatment, as assessed by trichrome and Sirius stain (1.64- and 1.01-fold increases over controls in PBS- and propranolol-treated groups, respectively; $P$ $=0.05$ for PBS group) (Figure 4A and Supplemental Figure 4B). Collagen genes identified by bioinformatics analysis were elevated in tumors from mice exposed to restraint stress, and these increases also were abrogated by propranolol (Supplemental Figure 4C). Similarly, restraint stress-induced increases in collagen were also abrogated by ADRB2 silencing, as shown by both trichrome and Sirius staining (Figure 4B and Supplemental Figure 4D). Treating animals with the nonspecific $\beta$-agonist or the ADRB2-specific agonist resulted in significant increases in tumoral collagen (1.81- and 1.75-fold increases for isoproterenol- and terbutaline-treated groups, respectively, over PBS-treated controls; $P$ $<0.05$ ) (Figure 4C). Moreover, NOF151 normal fibroblasts exposed to medium conditioned by NEtreated cancer cells showed similar elevations in the same collagen genes (Supplemental Figure 4E). However, NOF151 cells treated with conditioned media from ADRB2-silenced Skov3-ip1 cells did not show these elevations in collagen genes (Supplemental Figure 4F).

Induction of $C A F$ phenotype by NE-mediated inhibin $\beta A$ production. Our data demonstrate that tumorderived extracellular factors are important drivers of the CAF phenotype during chronic stress. Using a gene array data set of Skov3-ip1 and HeyA8 cells treated with NE, we identified several potential mediators 


\section{A}

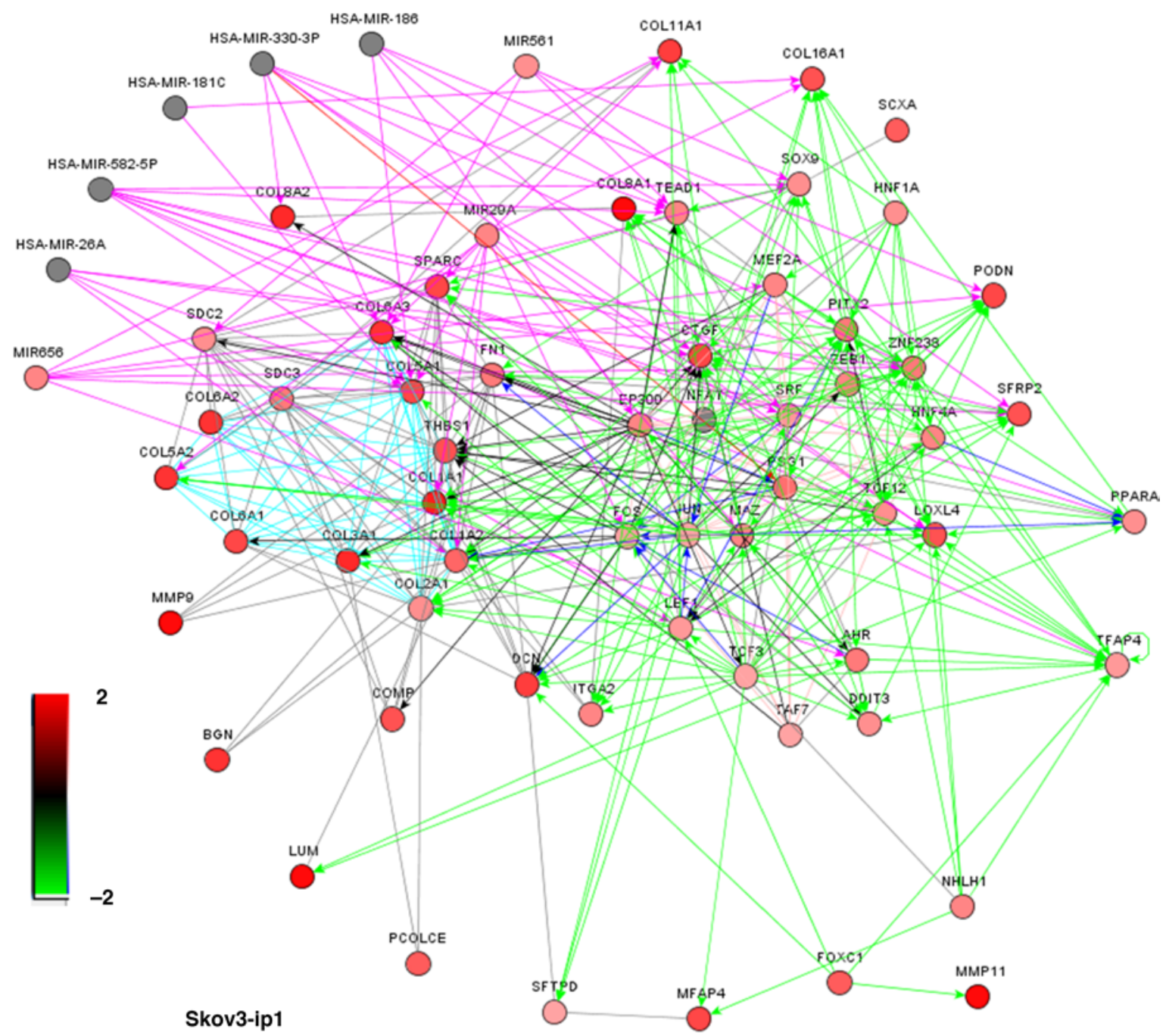

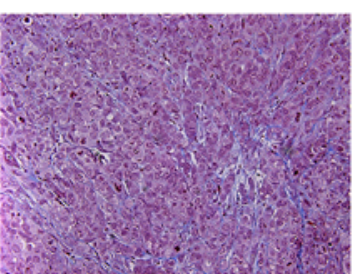

Control

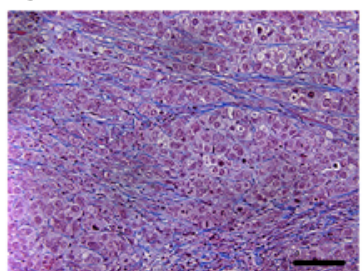

Stress

Figure 3. Adrenergic signaling increases collagen deposition and extracellular matrix formation in ovarian tumors. (A) An unbiased network of genes differentially upregulated in tumor samples from high depression score and low depression score patients. (B) Expression of collagen identified by Masson trichrome staining in micrographs of representative Skov3-ip1 tumors from control and restraint-stressed mice. Scale bars: $100 \mu \mathrm{m}$. $n=5 / \mathrm{group}$ for all data.

of this effect (11). To identify potential upstream regulators that are central to CAF activation in tumors, we used ingenuity pathway analysis (IPA) on gene arrays of microdissected CAFs and normal ovarian tissues to identify upstream regulators of CAFs in an independent data set (26). Combining the gene array and systems-based analyses, we identified IL6 (interleukin 6) and INBHA as factors that could potentially affect CAF biology (Figure 5A and ref. 11). We then used Oncomine to analyze coexpression of INHBA and IL6 with genes upregulated in ovarian cancer samples included in The Cancer Genome Atlas (TCGA). INHBA, but not IL6, was highly correlated with stroma-related genes that were upregulated in both patient data sets (Supplemental Tables 1 and 2). Therefore, we focused primarily on INHBA for subsequent experiments.

In the GEO data set GSE9116, we noted that INHBA mRNA levels were increased 2.14-fold in 
A
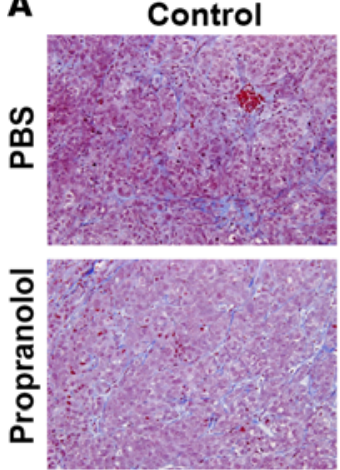

C

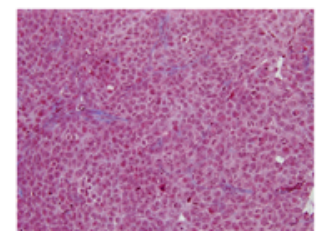

Stress
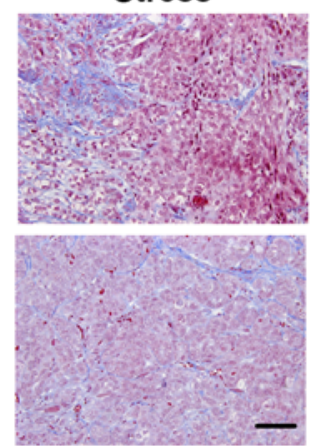

Isoproterenol
B
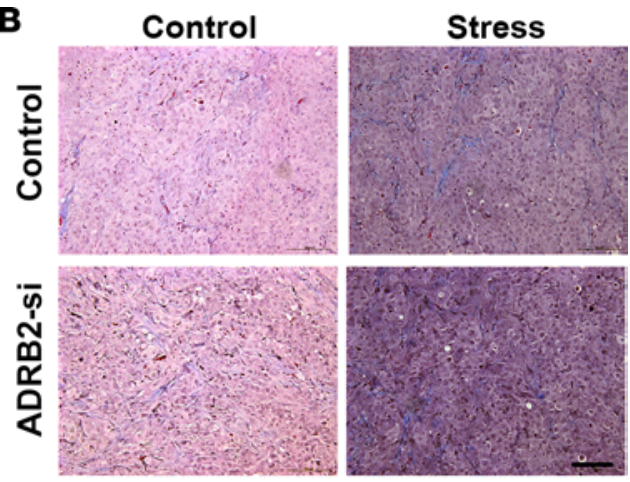

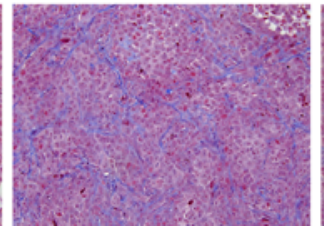

Terbutaline

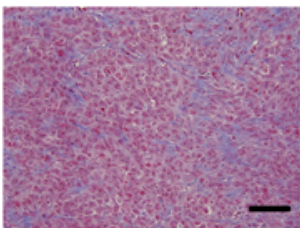

Figure 4. Adrenergic signaling increases collagen in an ADRB2-dependent manner. (A) Expression of collagen in representative Skov3-ip1 tumors from control and stressed mice treated with nonspecific $\beta$-blocker propranolol or phosphate-buffered saline solution (PBS, controls). (B) Expression of collagen identified by Masson trichrome staining in micrographs of representative HeyA8 tumors from control and stressed mice treated with control or ADRB2 siRNA. (C) Expression of collagen identified by Masson trichrome staining in micrographs of representative HeyA8 tumors from control and stressed mice treated with PBS (control), nonspecific $\beta$-agonist isoproterenol, or ADRB2-specific agonist terbutaline. Scale bars: $100 \mu \mathrm{m}$. $n=5 / \mathrm{group}$ for all data.

tumors from patients with high biobehavioral risk scores (19). Elevated INHBA levels were also associated with significantly shorter overall and progression-free survival in the TCGA ovarian cancer data set (Supplemental Figure 5A). Hazard ratios for collagens identified in the bioinformatics analysis were also analyzed for TCGA data (Supplemental Figure 5B). In the in vivo preclinical model, restraint stress significantly increased inhibin $\beta$ A levels in Skov3-ip1 and HeyA8 tumors (2.3- and 3.1-fold increases over non-stress controls, respectively, $P<0.05$ ) (Figure $5 \mathrm{~B}$ ). Treatment with propranolol abrogated the increases in tumoral INHBA at both protein and mRNA levels (Figure 5C and Supplemental Figure 5C).

In vitro, treatment of Skov3-ip1 cancer cells with NE significantly induced INHBA expression, and pretreatment of HeyA8 cells with propranolol or ADRB2-antagonist butoxamine abrogated this effect (Supplemental Figure 6, A and B). Silencing ADRB2 using siRNA decreased INHBA expression in Skov3-ip1 cells after NE treatment (Supplemental Figure 6C). Using TRANSFAC, a curated database for eukaryotic transcription factors, we identified CREB1 as a potential transcription factor driving INHBA expression. Chromatin immunoprecipitation analysis showed that NE increased CREB binding to the $I N H B A$ promoter by 4 -fold (Supplemental Figure 6D). Silencing $C R E B 1$ also reduced NE-induced increases in INHBA expression (Supplemental Figure 6E). Conditioned medium from CREB1-silenced Skov3-ip1 cells failed to increase ACTA2 expression in NOF151 cells, demonstrating the importance of inhibin $\beta$ A in driving the NE-mediated CAF phenotype (Supplemental Figure 6F).

We next sought to silence INHBA in cancer cells and validated several sequences of INHBA siRNA (Supplemental Figure 7, A and B). Conditioned medium from INHBA-silenced cancer cells also failed to increase ACTA2 levels in NOF151 cells (Supplemental Figure 7C). Furthermore, NOF151 fibroblasts exposed to medium conditioned by INHBA-silenced Skov3-ip1 cells also showed decreased levels of the same collagens that were elevated in the patient tumor samples (Supplemental Figure 7D).

Taken together, these results indicate that NE drives the CAF phenotype via an ADRB2/CREB/INHBA axis. To study the effects of inhibin $\beta \mathrm{A}$ in driving the CAF phenotype in vivo, we used a nanoliposomal (1,2-dioleoyl-sn-glycero-3-phosphatidylcholine [DOPC]) system to silence INHBA in the tumor cells in the HeyA8 model. Silencing INHBA decreased tumor growth and metastasis (Figure 5D). Gene knockdown was validated by RNA expression using human-specific sequences (Supplemental Figure 8A). More importantly, 

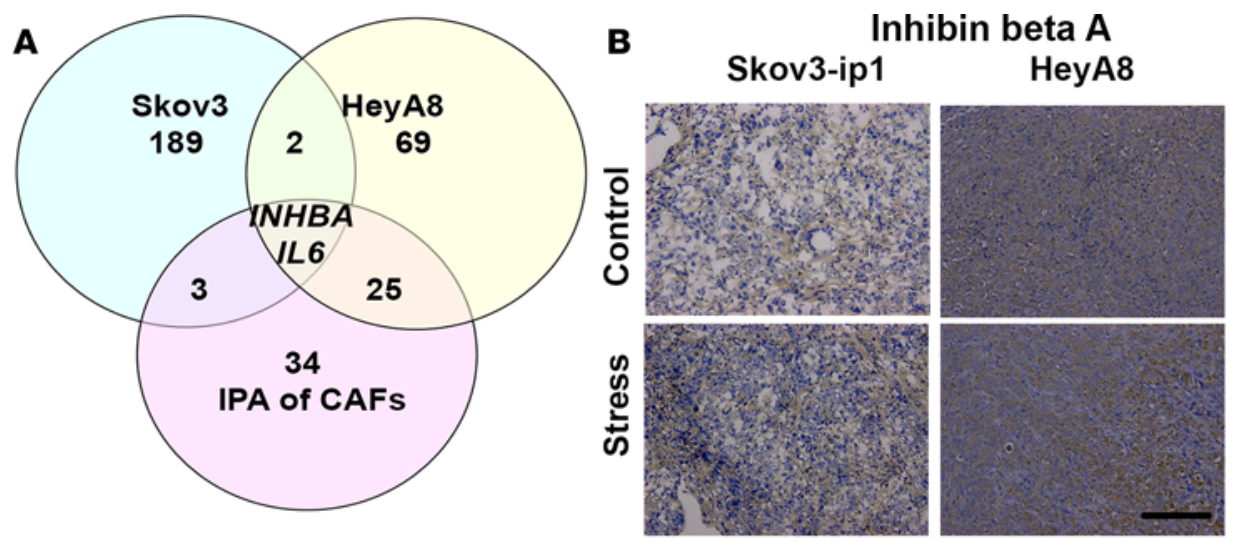

C Inhibin beta A
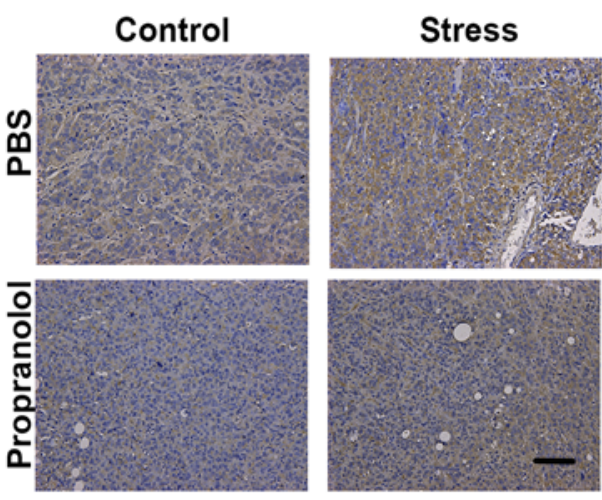

E $\alpha$-Smooth Muscle Actin
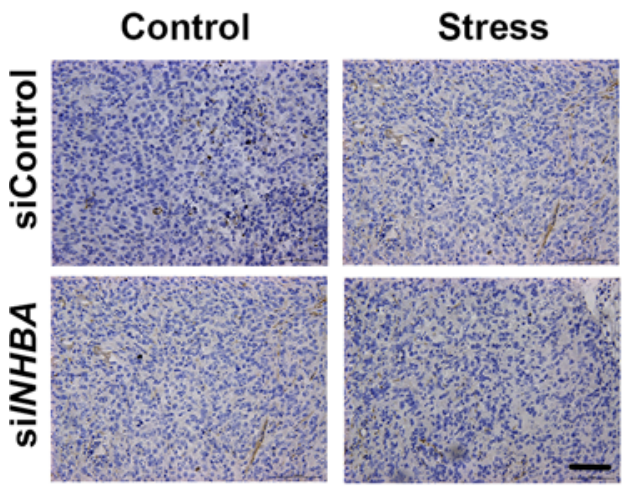

D

INHBA silencing in HeyA8 model
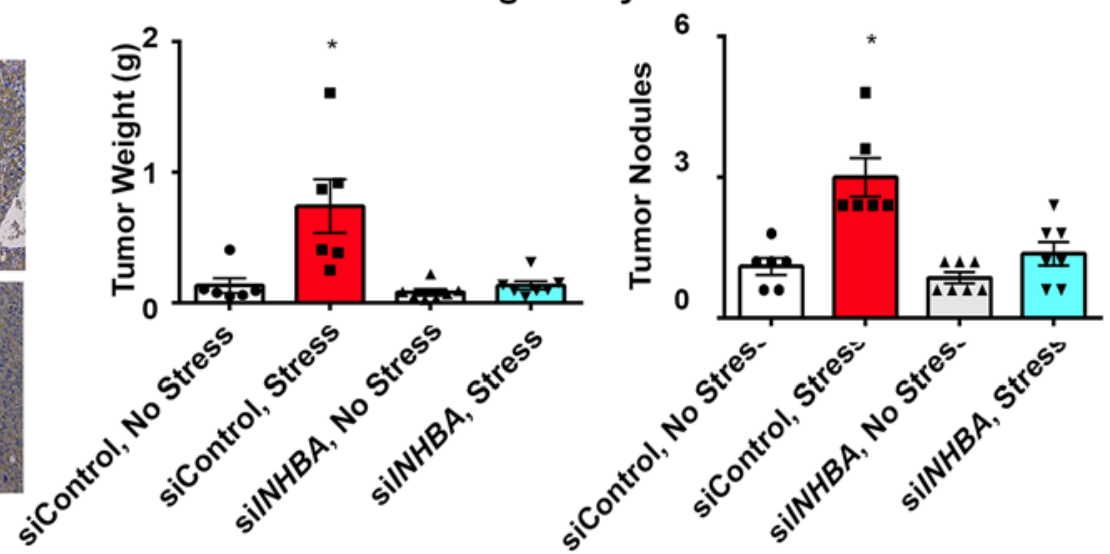

$\mathbf{F}$

Trichrome Staining

Control
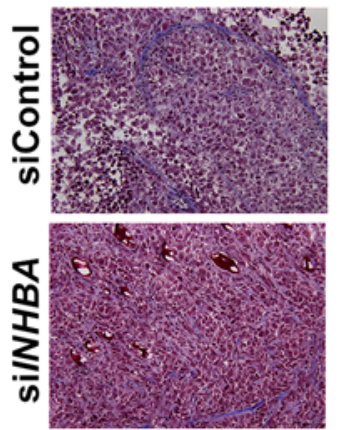
Stress
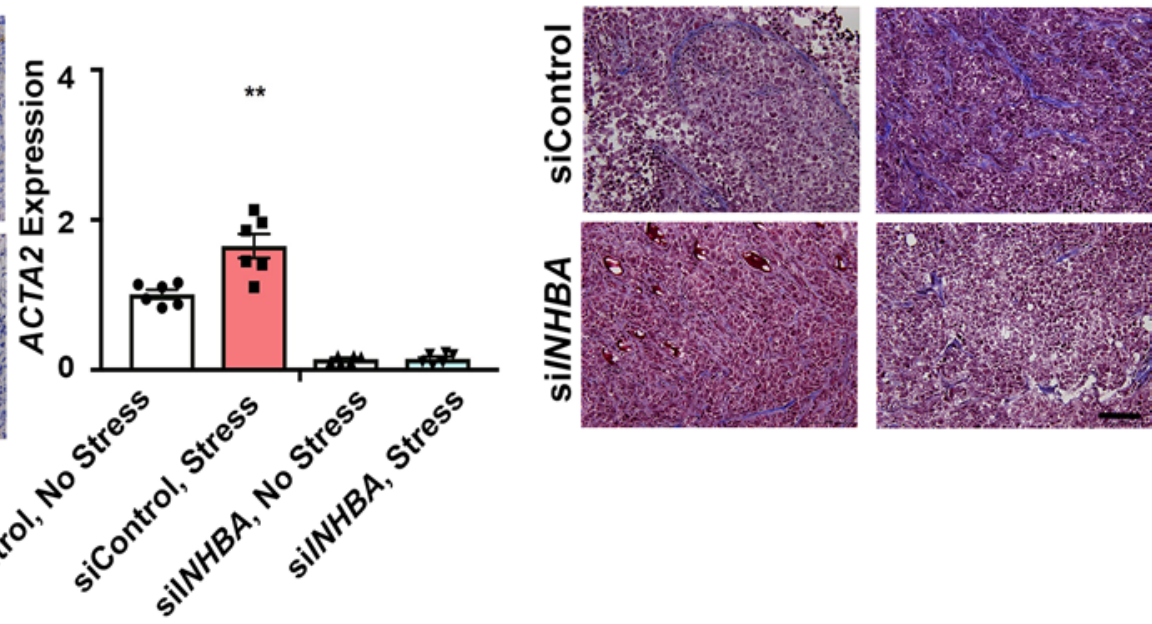

Figure 5. Induction of cancer-associated fibroblasts (CAFs) in ovarian carcinoma is mediated by inhibin $\boldsymbol{\beta}$ A downstream of norepinephrine. (A) Schema used for ingenuity pathway analysis (IPA) of different ovarian cancer cell lines to identify upstream regulators of CAF induction. (B) Expression of inhibin $\beta$ A in micrographs of representative Skov3-ip1 and HeyA 8 tumors from control and restraint-stressed mice. (C) Expression of inhibin $\beta$ A in micrographs of representative HeyA 8 tumors from control and stressed mice treated with nonspecific $\beta$-blocker propranolol or phosphate-buffered saline solution (PBS, control). (D) Effects of silencing INHBA in vivo on tumor weight and tumor nodules in HeyA8 tumor-bearing mice subjected to daily restraint stress and treated with either control siRNA or INHBA siRNA. $n=6$ or 7/group (1-way ANOVA for statistical significance). (E) Expression of CAF marker $\alpha$-smooth muscle actin ( $\alpha$-SMA) in micrographs and gene expression of representative HeyA 8 tumors from control and stressed mice treated with control or INHBA siRNA. (F) Expression of collagen identified by Masson trichrome staining in micrographs of representative HeyA8 tumors from control and stressed mice treated with control or INHBA siRNA. Scale bars: $100 \mu \mathrm{m}$. $n=5$ samples/group for all data and 1-way ANOVA for statistical significance. ${ }^{*} P<0.05,{ }^{*} P<0.01$. 
silencing inhibin $\beta$ A decreased restraint stress-mediated increases in the CAF phenotype at both protein and mRNA levels (Figure 5E and Supplemental Figure 8B). H\&E sections from tumors revealed increased stromal content in the control, stress group compared with the inhibin $\beta \mathrm{A}$-silenced groups (Supplemental Figure $8 \mathrm{C}$ ).

Silencing INHBA in HeyA8 tumor cells decreased collagen deposition in vivo and reduced levels of specific collagens that were significantly elevated in the patient tumor samples (Figure 5F and Supplemental Figure 8, D-F). To interrogate the signaling pathways operating in CAF activation, we used NOF151 fibroblasts. Inhibin $\beta$ A functions via ACVR2a or ACVR2b receptors and activates Smad2/3 proteins downstream. Tumor stroma was weakly positive for ACVR2a and positive for ACVR2b (Supplemental Figure 9A). Silencing ACVR2b, but not ACVR2a, in NOF151 cells abrogated changes in both ACTA2 and collagen expression mediated by NE-conditioned media (Supplemental Figure 9B).

Adrenergic signaling-mediated CAFs modulate collagen in breast and colorectal cancers. Clinical and preclinical data have shown that chronic stress can promote colorectal, breast, and prostate cancer progression in addition to ovarian cancer (1). Greater numbers of CAFs are associated with shorter survival, increased metastasis, and therapy resistance in these cancers $(14,15,28)$. To determine whether our findings apply to other cancer types, we examined both clinical data and in vivo tumor samples from breast and colon cancer models. In mouse models generated from ADRB-positive RKO colon cancer cells or GILM2 breast cancer cells, $\alpha$-SMA levels were significantly increased by restraint stress (2.2- and 3.4-fold increases for RKO and GILM2 models, respectively; $P<0.05$ ) (Figure 6A). There were concomitant increases in collagen in the tumors of mice subjected to restraint stress (1.7- and 2.1-fold increases for RKO and GILM2 models, respectively; $P<0.05$ ) (Figure 6B). Expression data from TCGA showed that INHBA levels are higher in tumors than in normal tissues (Figure 6C). Most intriguingly, analysis of genes that were coexpressed with INHBA in TCGA colorectal and ovarian cancers revealed significant overlap with the prominent CAF phenotype observed in ovarian cancer, and collagen patterns in these tumors were comparable to those in ovarian cancer (Supplemental Table 3 and Supplemental Figure 9, C and D). To further show the importance of chronic adrenergic signaling in promoting ECM- and CAF-related gene expression, we analyzed an independent set of renal carcinoma samples from patients with known CES-D scores for collagens and CAF markers. The results show higher levels of gene expression for collagens, INHBA, and ACTA2 in patients with higher CES-D scores (CES-D > 21 versus $<4)$. This finding did not reach statistical significance due to a limited number of samples (Figure 6D).

\section{Discussion}

In this study, one of the key findings is the prominent association between high biobehavioral risk and CAF phenotype. Utilizing both bioinformatics and molecular biology approaches, we identified a potentially novel mechanism of fibroblast activation triggered by inhibin $\beta$ A production by tumor cells in response to sustained adrenergic stimulation (Figure 7). We also show that downstream inhibin $\beta$ A signaling in CAFs drives levels of several collagens such as COL3A1, COL5A1, COL5A2, and COL11A1. Prior studies have identified the direct role of NE in driving molecular changes in tumor cells, but influences on stroma have been limited to natural killer cells, macrophage infiltration, and angiogenesis (1). Effects of adrenergic signaling on CAFs and ECM provide a previously unrecognized dimension that could have therapeutic implications. CAFs are the major part of tumor stroma and provide tumor cells with vital cues for invasion by remodeling the microenvironment through synthesis and deposition of collagens and producing proinflammatory cytokines and other growth factors (29). Collagens in the ECM can engage integrins on tumor cells, impede $\mathrm{T}$ cell infiltration, and facilitate invasion and metastasis. Importantly, collagens identified in our study (e.g., COL5A1, COL11A1, and COL1A1) are also associated with increased metastasis and poor overall survival in ovarian cancer (30)

We demonstrated that NE-ADRB2-CREB-mediated inhibin $\beta$ A production in tumor cells is important for induction of CAF phenotype in tumors. NE-mediated ADRB2 and CREB signaling in cancer cells is important for increasing angiogenesis, tumor invasion, and inflammation. Inhibin $\beta \mathrm{A}$ is a member of the TGF- $\beta$ pathway and is overexpressed in prostate, ovarian, colorectal, and breast cancers (31-33). Elevated serum and plasma levels of inhibin $\beta$ A have been reported in patients with metastatic ovarian, breast, and prostate cancers (31-33). Inhibin $\beta$ A is shown to have other effects on tumors such as promoting stem cell phenotype and modulating cancer cachexia (34). High levels of INHBA are also associated with poor clinical outcome of ovarian cancer patients in TCGA data. A major finding of this study is the demonstration that inhibin $\beta \mathrm{A}$ is a driver of the CAF phenotype in ovarian cancer. Several collagens are also coexpressed 
A
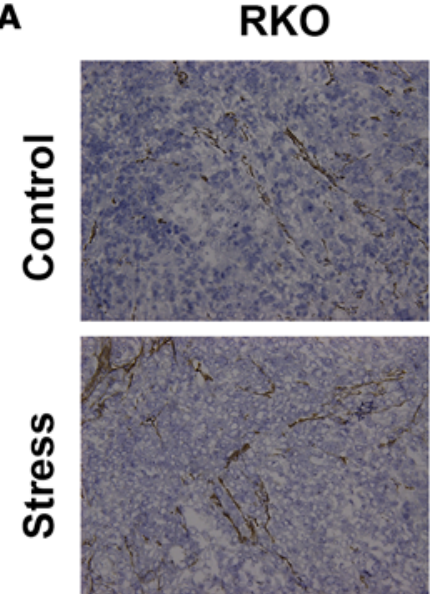

GILM2
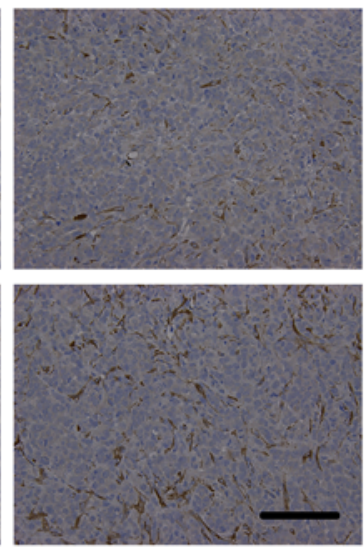

B
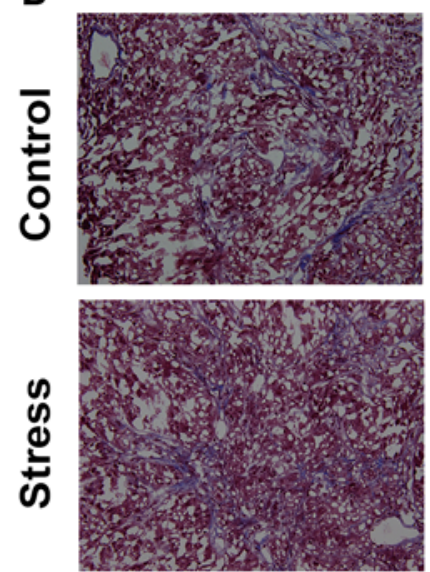

GILM2
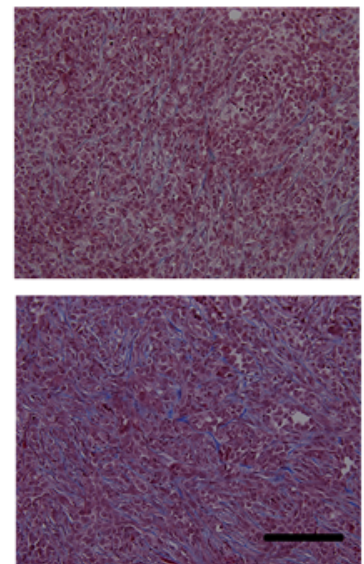

C

INHBA differential plot

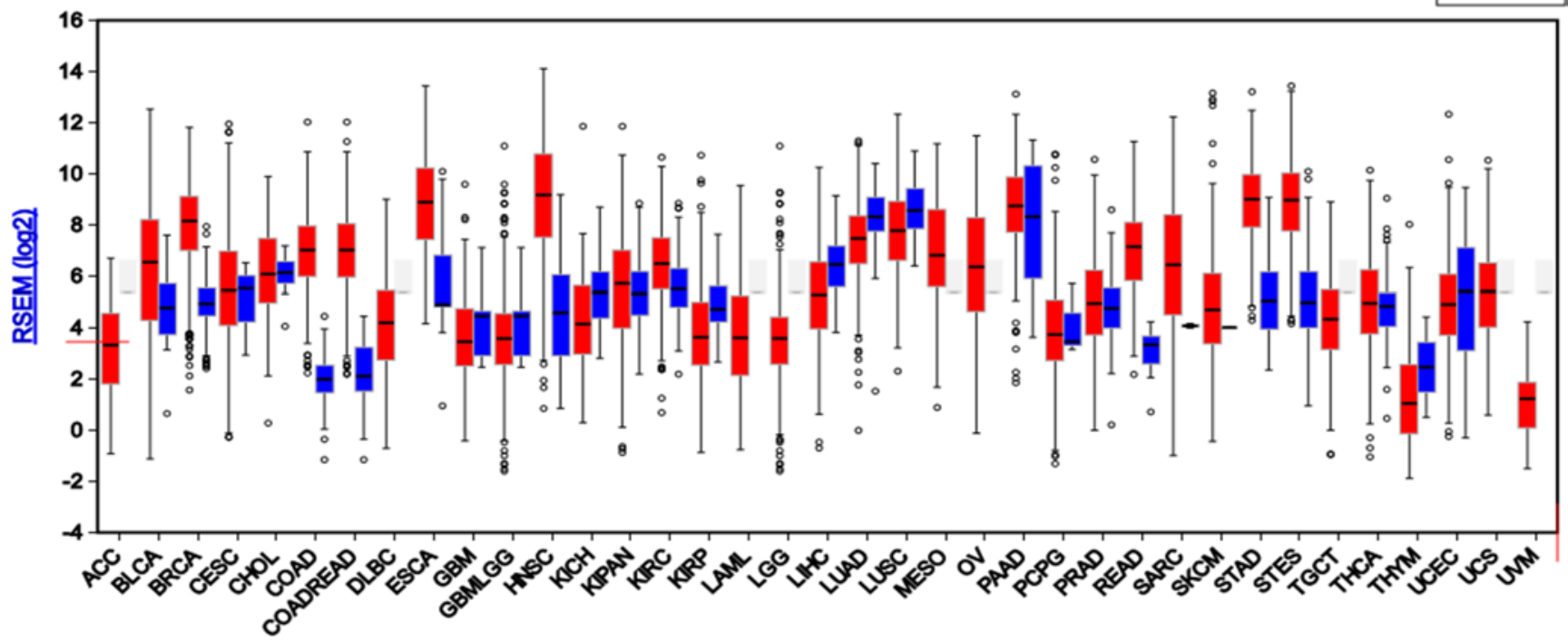

D

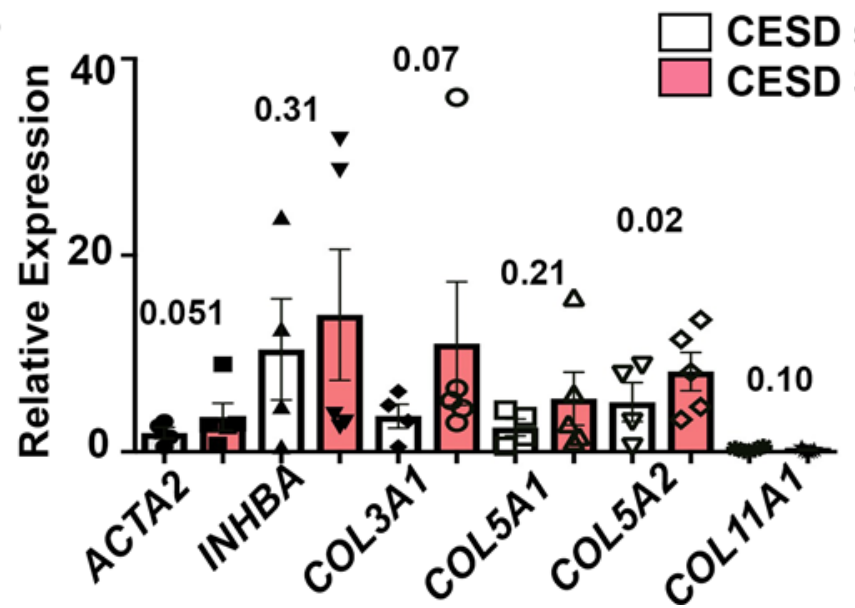

Figure 6. Restraint stress induces cancer-associated fibroblasts (CAFs) in colon, breast, and renal cancer models. (A) Expression of CAF marker $\alpha$-smooth muscle actin ( $\alpha$-SMA) in micrographs of representative tumors from control and restraint-stressed mice in adrenergic receptor-positive RKO (colon cancer) and GILM2 (breast cancer) models. (B) Expression of collagen identified by Masson trichrome staining in micrographs of representative RKO and GILM2 tumors from control and stressed mice. (C) Pan-cancer INHBA expression from TCGA data. (D) Clinical validation of adrenergic-mediated CAF phenotype in renal cell carcinoma samples using qRT-PCR. $P$ values as indicated, determined by 2-tailed Student's $t$ test ( 9 total samples: 4 in Center for Epidemiologic Studies Depression Scale [CES-D] < 4 and 5 in CES-D > 21). Scale bars: $100 \mu \mathrm{m}$. $n=5 /$ group for RKO data and $n=3 /$ group for GILM2 data. 


\section{Adrenergic Signaling}
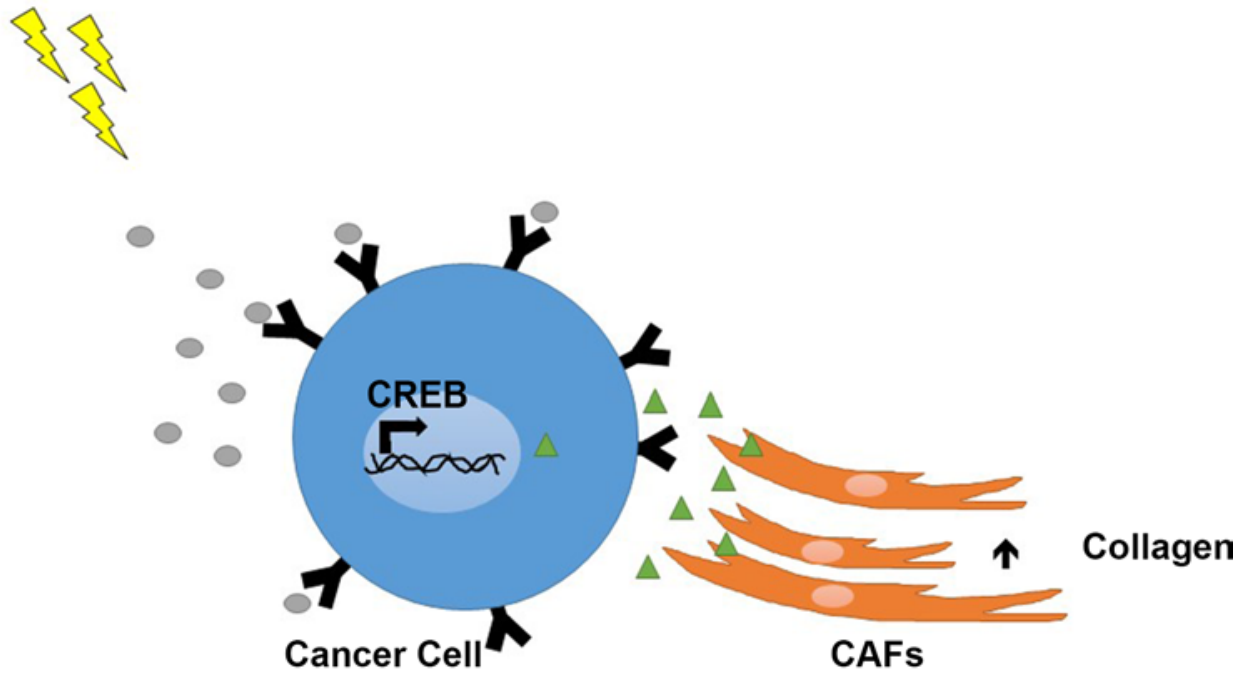

- Norepinephrine $\Delta$ Inhibin beta A

Y ADRB2

Figure 7. Proposed model of cancer-associated fibroblast (CAF) activation and increased collagen deposition in response to adrenergic signaling in ovarian carcinoma. CREB, CAMP responsive element binding protein; ADRB2, $\beta_{2}$ adrenergic receptor.

significantly with INHBA in ovarian, breast and colon tumors using TCGA data. Although coexpression of collagen genes with $I N H B A$ has been reported in some cancers, the functional relationships have not been well understood (35). To our knowledge, this is the first report to show that inhibin $\beta \mathrm{A}$ is necessary for increased collagen levels in CAFs and may be an important biomarker for tumor progression. In our study, silencing inhibin $\beta \mathrm{A}$ in tumor cells reduced the levels of adrenergic-stimulated CAFs and collagen significantly. Several studies have focused on targeting inhibin $\beta$ A to prevent cancer cachexia, but our results demonstrate important roles in modulating the tumor stroma (36-38). Our results, therefore, also have important therapeutic implications, as compounds that inactivate or deplete inhibin $\beta$ A signaling might be effective in tumors under chronic adrenergic stimulation. STM 434, a humanized decoy receptor for the activin A receptor, is currently in a clinical trial for treating patients with metastatic and chemotherapyresistant ovarian cancer (NCT02262455). $\beta$-Blockers have been shown to be associated with survival benefit in several cancers in retrospective studies; our results suggest that $\beta$-blockers could be useful in combination with novel drugs such as inhibin $\beta$ A decoy receptors. Disruption of interactions between cancer cells and stress hormones or cancer cells and fibroblasts may improve outcomes of cancer patients.

\section{Methods}

\section{Cell lines}

Skov3-ip1, A2780-ip2, and HeyA8 ovarian cancer cell lines have been described previously (11). SKOV3ip1, HeyA8, and A2780-ip2 were obtained from the Cancer Center Support Grant-funded (CCSG-funded) Characterized Cell Line Core Facility at MD Anderson Cancer Center (Houston, Texas, USA). STR DNA fingerprinting was done by the CCSG-funded Characterized Cell Line Core (NCI CA016672). They were maintained in RPMI-1640 medium supplemented with $15 \%$ fetal bovine serum and $0.1 \%$ gentamicin sulfate at $37^{\circ} \mathrm{C}$. ID8-ip1 cells were a gift from George Coukos (Ludwig Institute for Cancer Research, Zurich, Switzerland) and maintained in high-glucose DMEM supplemented with $10 \%$ fetal bovine serum, $0.1 \%$ gentamicin, and $0.1 \%$ insulin-transferrin sulfate (39). NOF151 normal ovarian fibroblasts, whose derivation has been described before $(26,40)$, were a gift from Jinsong Liu. These cells were maintained in a 1:1 mixture of Medium 199 and MCDB 105 medium supplemented with $10 \%$ fetal bovine serum, $1 \%$ penicillin-streptomycin, and $5 \mu \mathrm{g}$ epidermal growth factor. All cells were grown at $37^{\circ} \mathrm{C}$ in a humidified 
chamber with $5 \% \mathrm{CO}_{2}$.

For all in vitro experiments, cancer cells at $70 \%$ confluence were serum starved overnight before treatment. NE (10 $\mu \mathrm{M}$; Sigma-Aldrich) was dissolved in sterile water just before it was added to the cells. Isoproterenol and terbutaline (Sigma-Aldrich) were used at published concentrations $(6,8)$. Propranolol and butoxamine were purchased from Tocris and used at published concentrations $(6,8)$. Treatment with an antagonist, if pertinent, began 1 hour before NE was added to the cell culture medium. Treatment with agonists was started at the same time as NE treatment. Genes were silenced by using a reverse-transfection protocol with RNAimax and control siRNA (sequence: UUAUGCCGAUCGCGUCACA), specific human INHBA siRNA (sequence: CCAACAGGACCAGGACCAA), human ADRB2 siRNA (sequence: GCCATTACTTCACCTTTCA), human ACVR2a siRNA (sequence: GCUCCAACCUCGAAGUAGA) and human ACVR2b siRNA (sequence: CUCGACUUUGGGUUGGCCUU) according to the manufacturer's (SigmaAldrich) recommendations. Briefly, $40 \mathrm{~nm}$ siRNA was mixed with RNAimax transfecting agent and added to culture medium for 4 hours. Fresh complete medium was added and cells were allowed to grow. Silencing was assessed at 48 hours using quantitative reverse transcription PCR (qRT-PCR) analysis.

\section{Chronic stress model}

All animal experiments were approved by the Institutional Animal Care and Use Committee at The University of Texas MD Anderson Cancer Center, where the experiments were carried out.

Experiments involving human cancer cell- and murine cancer cell-derived mouse tumor models were performed in 8- to 12-week-old female athymic nude and C57/B6 mice, respectively, obtained from Taconic Farms. Adrenalectomized mice were received from Taconic Farms 3 days after surgery and were given an additional 10 days to recover before restraint stress started. Chronic stress was induced experimentally by using a restraint-stress procedure that has been previously described (20). In brief, mice were enclosed in a movement-restricted space for 2 hours daily for the duration of the experiment.

Tumor cells were injected intraperitoneally into mice in all groups 1 week after the stress procedure began (Skov3-ip1: 1 million cells/animal; HeyA8: 250,000 cells/animal; ID8-ip1: 2 million cells/animal). Mice were randomly assigned to groups ( $n=10$ /group), and siRNA ( $3.5 \mu \mathrm{g}$ in DOPC nanoliposomes) treatment was started 5 days after tumor cell injection and continued twice weekly for the duration of the experiment. Mice were treated by intraperitoneal injection with propranolol or a specific $\beta$-blocker or adrenergic agonist daily for the duration of the experiment. The animals were sacrificed by cervical dislocation when they became moribund and examined for visible disease, and mouse weight, tumor weight, and number and distribution of nodules were recorded.

\section{Immunohistochemistry}

Both frozen and paraffin sections were used for immunohistochemical analyses. Paraffin sections were heated for 20 minutes at $56^{\circ} \mathrm{C}$ and were deparaffinized in xylene and dehydrated/rehydrated in decreasing grades of alcohol and PBS. Antigen retrieval was performed in citrate buffer $(\mathrm{pH} \mathrm{6.0)}$ in the steamer for 30 minutes. Frozen sections were fixed in acetone and acetone/chloroform. After endogenous peroxidase blocking with hydrogen peroxide in methanol and 3 washes with PBS, the slides were incubated with primary antibodies $\alpha$-SMA (1:100, ab5694, Abcam), FAP (1:100, AF3715, R\&D Systems), inhibin $\beta$ A (1:100, ab56057, Abcam), desmin (1:100, ab15200, Abcam), collagen 3 (1:100, ab7778, Abcam), collagen 5 (1:50, ab7046, Abcam), ACVR2a (1:100, PA5-13886, Thermo Fisher Scientific), ACVR2b (1:100, ab135635, Abcam) overnight at $4^{\circ} \mathrm{C}$. Sections were exposed to matching secondary antibodies (Jackson ImmunoResearch) for 1 hour at room temperature and staining was developed using 3,3'-diaminobenzidine. Nuclei were stained with hematoxylin. Quantification of proteins was done by analyzing both the proportion and intensity and presented as fold-change normalized to control.

\section{Migration assay}

Migration of NOF151 was measured in vitro. Inserts ( $8 \mu \mathrm{m}$; Millipore) were coated with $1 \%$ gelatin for migration assays. A total of 50,000 cells were placed in each upper well and allowed to move toward conditioned medium in the lower chamber in the presence or absence of NE. Migration was assessed 6 hours after treatment by fixing the cells in Protocol Hema3 (Thermo Fisher Scientific). Cells were counted in 5 randomly chosen high-power fields, and cell counts are reported as average numbers of cells migrated. 


\section{Collagen staining}

Collagen distribution and density were assessed by using Masson trichrome staining (Abcam) and Sirius staining (Chrondrex) for both paraffin and frozen slides. Paraffin sections were deparaffinized and dehydrated/rehydrated in alcohol and distilled water, and frozen slides were dried at room temperature followed by fixation in formalin for 30 minutes. Slides were then fixed in preheated Bouin Solution (Sigma-Aldrich). Slides were washed in tap water until the water ran clear, and Abcam's staining protocol was followed. Quantification of collagens was done by analyzing both the proportion and intensity and presented as fold change normalized to control. Nuclei were stained with Weigert iron hematoxylin. Sirius Red/Fast Green Collagen staining for collagens 1 and 3 was done by using a kit (Chondrex).

\section{qRT-PCR}

Total RNA was extracted from cells by using the Zymo Research RNA isolation kit with TRIzol reagent (Invitrogen) according to the manufacturer's standard protocol. Complementary DNA was synthesized from $1 \mu \mathrm{g}$ of total RNA using the Verso cDNA Synthesis kit (Thermo Fisher Scientific) according to the supplier protocol, using random hexamers and oligo-dT primers in a 3:1 ratio. Quantitative PCR was performed using SYBR Green Master Mix on the 7500 Real-Time PCR System (Applied Biosystems) and standard protocols. The primer sequences used are included in Supplemental Table 4.

\section{Proteome profiler}

NOF151 cells were exposed to serum-free medium, medium conditioned by untreated cancer cells, or medium conditioned by NE-treated cancer cells. The conditioned media were spun down at $200 \mathrm{~g}$ to remove cell debris. Total protein from each culture was quantified according to Bradford protein assay, and $250 \mu \mathrm{g}$ of each protein sample was assayed in parallel using a Human Cytokine Array kit (R\&D Systems), according to the manufacturer's protocol.

\section{Inhibin $\beta$ A ELISA}

The inhibin $\beta$ A ELISA kit was obtained from MyBiosource. Supernatants from HeyA 8 cells treated with $\mathrm{NE}$ or a specific $\beta$-blocker, and with control or INHBA siRNA, were collected and spun down to remove cell debris. Supernatants were immediately stored at $-80^{\circ} \mathrm{C}$ until use. The ELISA kit was used per the manufacturer's instructions. In short, a plate was incubated with standards or 1:10 diluted supernatants for 2 hours. The plate was then incubated with biotin-labeled antibody for 1 hour. After the plate was washed with PBS, it was developed with colorimetric reagent and read at $540 \mathrm{~nm}$. Results are expressed as pg protein/million cells.

\section{Clinical samples}

Renal cell carcinoma. Fresh renal cell carcinoma tumor samples were obtained during surgery. In short, a minimum of 2 grams of tumor was obtained and processed from each patient by a surgical pathologist who immediately dissected the specimen in a sterile fashion. The tumor was placed in RPMI 1640 medium, cut into 1-mm pieces, and minced using sterile forceps and a sterile scalpel. The digest was washed through a mesh to obtain a single-cell suspension, and viable cells counted. The vials were maintained overnight at $-80^{\circ} \mathrm{C}$ and then transferred to liquid nitrogen. The MD Anderson IRB approved the protocols for handling and analyzing the specimens. Patients provided written informed consent for the collection of specimens and clinical data in accordance with the ethics guidelines of and with approval from the MD Anderson IRB (41). Patients also completed several psychosocial questionnaires given by the Department of Behavioral Science at MD Anderson Cancer Center. The CES-D was used to assess depressive symptoms, with scores of 16 or above classified as meeting the screening criteria for depressive symptoms. Samples from 4 patients with low CES-D scores (CES-D score $<4 ; 2$ females and 2 males) and 5 patients with high CES-D scores (CES-D score $>21 ; 3$ males and 2 females), matched for having stage 2 or 3 disease, were chosen for analysis.

Ovarian cancer. Fresh tumor samples were obtained from surgery and frozen in liquid nitrogen within approximately 30 minutes following surgical removal from the patient. Tumor $(0.1 \mathrm{~g})$ was processed for total RNA, and genome-wide transcription analyzed using Affymetrix U133A high-density oligonucleotide arrays. Total RNA was extracted and treated with DNAse (Qiagen) following the manufacturer's protocol to remove DNA impurities (followed by 1 wash in RW1 reagent and 1 wash in RPE reagent to remove excess salt). An 
Agilent 2100 bioanalyzer was used to analyze RNA purity and integrity and Affymetrix U133A array was used for gene expression in the UCLA DNA Microarray Core, as previously described (19). IRBs at the University of Iowa, the University of Miami, and the University of California at Los Angeles approved the protocols. For this data set, psychosocial data were obtained from patients undergoing surgical resection of ovarian carcinoma. Patients completed the CES-D (42) and Social Provisions Scale (SPS) (43) at home between their initial clinic visit and their surgery. High biobehavioral risk was determined by scores of 16 or higher on the CES-D and an SPS attachment subscale score below 15 (the median value). Tumors from 5 patients with high and 5 with low biobehavioral risk were matched for stage, grade, and histological subtype.

TCGA data. Primary ovarian cancer samples from TCGA were used to generate survival graphs using an online tool (44). In short, this tool uses gene expression data (Affymetrix platform) from 522 patients to generate overall and progression-free survival using the probe 210511_s_at for INHBA. The samples were split by the median value and best cutoff was selected automatically. For survival analyses, the tumor types of interest are breast (BRCA), OV, colon, and rectum. General plots showing the hazard ratio, confidence interval, and $P$ value for each case were generated for the entire data set.

\section{Bioinformatics analysis}

Netwalker. NetWalker (https://netwalkersuite.org/) was used to analyze whole-tumor gene expression data from ovarian cancer patients with known biobehavioral risk scores (GSE9116), calculated as above. Using Netwalker we developed unbiased networks of upregulated and downregulated genes. Analyses were run considering the ratio (average of high biobehavioral risk/low biobehavioral risk). The network with the highest scoring interactions throughout was identified, along with the functions of the genes involved.

$I P A$. Significantly upregulated genes (fold-change $>2$ ) for whole-tumor gene expression data from ovarian cancer patient with known biobehavioral risks (GSE9116) were compared with those identified by comparing microdissected CAFs with normal fibroblasts from ovarian tissue (GSE40643). Common genes that were upregulated in both settings were identified. IPA was used to identify potential upstream regulators of CAFs. The genes identified were then compared to a gene array of Skov3-ip1 and HeyA8 cells that were treated with NE to identify potential regulators that can be activated by adrenergic stimulation.

Oncomine. To identify genes that are significantly correlated with INHBA, we analyzed the TCGA data sets for ovarian, colorectal, and breast cancers using Oncomine (https://www.oncomine.org/). Significantly coexpressed genes from ovarian, breast, and colorectal cancers were identified and tabulated.

RNA-Seq data. From the Level 3 (public) data from TCGA, including values from RNA sequencing (RNA-Seq) assays, we generated plots that included summary values for INHBA from "RNA-SeqV2" across all tumor types, including all samples for which these data were available. The samples are ordered by tissue type.

\section{Statistics}

MS Excel or GraphPad Prism software was used to analyze data. Either 2-tailed Student's $t$ test or analysis of variance (ANOVA), and the Mann-Whitney test were used to compare differences between continuous variables. We determined that, using 2-way ANOVA, a sample size of 10 animals per group would provide an effective size of 1.3 with $80 \%$ power at a significance of $P$ equals 0.05 . We considered $P$ less than 0.05 to be significant. All statistical analysis results were expressed as mean \pm SEM.

\section{Study approval}

The Institutional Animal Care and Use Committee at The University of Texas MD Anderson Cancer Center (Houston, Texas), where the experiments were carried out, reviewed and approved all animal experiments. The MD Anderson IRB (Houston, Texas) reviewed and approved the protocols for handling and analyzing the renal cell carcinoma specimens. Patients provided written informed consent for the collection of specimens and clinical data in accordance with the ethics guidelines of and with approval from the IRB at MD Anderson. IRBs at the University of Iowa (Iowa City, Iowa), the University of Miami (Miami, Florida), and the University of California at Los Angeles (Los Angeles, California) approved the protocols for collection of ovarian tumors from patients and written consent was provided by all patients in the study. 


\section{Author contributions}

ASN and AKS conceived the project and designed the experiments. ASN, GAP, KMG, JKA, NCS, LSM, NBJ, YK, SP, SYW, YL, and MH carried out or participated in in vitro and in vivo experiments. CRA and GLB designed and prepared nanoliposomes for in vivo studies. JL and SCM provided normal ovarian fibroblast cell line used for in vitro studies. LC provided the renal carcinoma samples. YW and $\mathrm{KB}$ analyzed RNA-Seq data from TCGA samples across cancer types. PR analyzed patient gene arrays with NetWalker for protein interaction analysis. ASN, RLD, SYW, and CI designed and performed computational analyses. SKL and LC contributed to assessment of psychosocial factors and patient data analysis. ASN wrote the manuscript. ASN, SKL, LC, SWC, and AKS participated in manuscript preparation. All authors edited and approved the final manuscript.

\section{Acknowledgments}

We thank the following for funding support to AKS: the NIH (CA116778, CA104825, CA109298, CA193249 and CA140933, P50CA083639, P50CA098258, AG017265, AG033590); the RGK Foundation; the American Cancer Society Research Professor award; the Blanton-Davis Ovarian Cancer Research Program; and the Frank McGraw Memorial Chair in Cancer Research. SWC is supported by NIH grants CA116778, AG017265, and AG033590. AN is supported in part by the CPRIT Graduate Scholar Fellowship (RP140106). KMG is supported Altman-Goldstein Discovery fellowship. SYW is supported by Ovarian Cancer Research Fund Alliance, Foundation for Women's Cancer, Colleen Dream Foundation, and CPRIT training grants (RP101502 and RP101489). RLD is supported by the NCI-DHHS-NIH T32 training grant (T32 CA101642). MH is supported by a fellowship of the Deutsche Forschungsgemeinschaft (DFG). YAL is supported by a training fellowship from the Keck Center of the Gulf Coast Consortia, on the Training Program in Biomedical Informatics, National Library of Medicine (NLM) T15LM007093 (PI, Lydia E. Kavraki) and by P50 CA083639. SP is supported by a Foundation for Women's Cancer grant. This research was supported in part by NCI grant R01CA090966 (PI, Lorenzo Cohen), by NCI grants CA193249 and CA140933 (PI, SKL), NCI Core grant CA016672 awarded to the MD Anderson Cancer Center, and the Richard E. Haynes Distinguished Professorship in Clinical Cancer Prevention. We would also like to thank Kathryn Hale at MD Anderson for help with scientific editing.

Address correspondence to: Anil K. Sood, 1220 Holcombe Blvd No. 1260, Houston, Texas 77030, USA. Phone: 713.792.4130; Email: asood@mdanderson.org.

GAP's present address is: Department of Basic Sciences, Division of Pharmacology, Ponce Health Sciences University and Division of Cancer Biology, Ponce Research Institute, Ponce, Puerto Rico.

YK's present address is: Shanghai Key Laboratory of Female Reproductive Endocrine-Related Diseases, Obstetrics and Gynecology Hospital of Fudan University, Shanghai, China.

1. Cole SW, Nagaraja AS, Lutgendorf SK, Green PA, Sood AK. Sympathetic nervous system regulation of the tumour microenvironment. Nat Rev Cancer. 2015;15(9):563-572.

2. De Giorgi V, et al. $\beta$-Adrenergic-blocking drugs and melanoma: current state of the art. Expert Rev Anticancer Ther. 2012;12(11):1461-1467.

3. Lemeshow S, et al. Beta-blockers and survival among Danish patients with malignant melanoma: a population-based cohort study. Cancer Epidemiol Biomarkers Prev. 2011;20(10):2273-2279.

4. Obeid EI, Conzen SD. The role of adrenergic signaling in breast cancer biology. Cancer Biomark. 2013;13(3):161-169.

5. Partecke LI, et al. Chronic stress increases experimental pancreatic cancer growth, reduces survival and can be antagonised by beta-adrenergic receptor blockade. Pancreatology. 2016;16(3):423-433.

6. Kang Y, et al. Adrenergic stimulation of DUSP1 impairs chemotherapy response in ovarian cancer. Clin Cancer Res. 2016;22(7):1713-1724.

7. Masur K, Niggemann B, Zanker KS, Entschladen F. Norepinephrine-induced migration of SW 480 colon carcinoma cells is inhibited by beta-blockers. Cancer Res. 2001;61(7):2866-2869.

8. Nagaraja AS, et al. Sustained adrenergic signaling leads to increased metastasis in ovarian cancer via increased PGE2 synthesis. Oncogene. 2016;35(18):2390-2397.

9. Rosenne E, et al. In vivo suppression of NK cell cytotoxicity by stress and surgery: glucocorticoids have a minor role compared to catecholamines and prostaglandins. Brain Behav Immun. 2014;37:207-219.

10. Shahzad MM, et al. Stress effects on FosB- and interleukin-8 (IL8)-driven ovarian cancer growth and metastasis. $J$ Biol Chem. 2010;285(46):35462-35470. 
11. Armaiz-Pena GN, et al. Src activation by $\beta$-adrenoreceptors is a key switch for tumour metastasis. Nat Commun. $2013 ; 4: 1403$.

12. Sood AK, et al. Adrenergic modulation of focal adhesion kinase protects human ovarian cancer cells from anoikis. J Clin Invest. 2010;120(5):1515-1523.

13. Erez N, Truitt M, Olson P, Arron ST, Hanahan D. Cancer-associated fibroblasts are activated in incipient neoplasia to orchestrate tumor-promoting inflammation in an NF-кB-dependent manner. Cancer Cell. 2010;17(2):135-147.

14. Kalluri R, Zeisberg M. Fibroblasts in cancer. Nature Rev Cancer. 2006;6(5):392-401.

15. Madar S, Goldstein I, Rotter V. 'Cancer associated fibroblasts' - more than meets the eye. Trends Mol Med. 2013;19(8):447-453.

16. Mitra AK, et al. MicroRNAs reprogram normal fibroblasts into cancer-associated fibroblasts in ovarian cancer. Cancer Discov. 2012;2(12):1100-1108.

17. Ryner L, et al. Upregulation of periostin and reactive stroma is associated with primary chemoresistance and predicts clinical outcomes in epithelial ovarian cancer. Clin Cancer Res. 2015;21(13):2941-2951.

18. Yeung TL, Leung CS, Li F, Wong SS, Mok SC. Targeting stromal-cancer cell crosstalk networks in ovarian cancer treatment. Biomolecules. 2016;6(1):3.

19. Lutgendorf SK, et al. Depression, social support, and beta-adrenergic transcription control in human ovarian cancer. Brain Behav Immun. 2009;23(2):176-183.

20. Thaker PH, et al. Chronic stress promotes tumor growth and angiogenesis in a mouse model of ovarian carcinoma. Nat Med. 2006;12(8):939-944.

21. Ozdemir BC, et al. Depletion of carcinoma-associated fibroblasts and fibrosis induces immunosuppression and accelerates pancreas cancer with reduced survival (vol 25, pg 719, 2014). Cancer Cell. 2015;28:831-833.

22. Sugimoto H, Mundel TM, Kieran MW, Kalluri R. Identification of fibroblast heterogeneity in the tumor microenvironment. Cancer Biol Ther. 2006;5(12):1640-1646.

23. Kvetnansky R, Sun CL, Lake CR, Thoa N, Torda T, Kopin IJ. Effect of handling and forced immobilization on rat plasma levels of epinephrine, norepinephrine, and dopamine-beta-hydroxylase. Endocrinology. 1978;103(5):1868-1874.

24. Hassan S, et al. Behavioral stress accelerates prostate cancer development in mice. J Clin Invest. 2013;123(2):874-886.

25. Magnon C, et al. Autonomic nerve development contributes to prostate cancer progression. Science. 2013;341(6142):1236361.

26. Leung CS, et al. Calcium-dependent FAK/CREB/TNNC1 signalling mediates the effect of stromal MFAP5 on ovarian cancer metastatic potential. Nat Commun. 2014;5:5092.

27. Karagiannis GS, et al. Collective migration of cancer-associated fibroblasts is enhanced by overexpression of tight junctionassociated proteins daudin-11 and occludin. Mol Oncol. 2014;8(2):178-195.

28. Micke P, Ostman A. Tumour-stroma interaction: cancer-associated fibroblasts as novel targets in anti-cancer therapy? Lung Cancer. 2004;45(supp1 2):S163-S175.

29. Kalluri R. The biology and function of fibroblasts in cancer. Nat Rev Cancer. 2016;16(9):582-598.

30. Cheon DJ, et al. A collagen-remodeling gene signature regulated by TGF- $\beta$ signaling is associated with metastasis and poor survival in serous ovarian cancer. Clin Cancer Res. 2014;20(3):711-723.

31. Leto G, et al. Activin A circulating levels in patients with bone metastasis from breast or prostate cancer. Clin Exp Metastasis. 2006;23(2):117-122.

32. Reader KL, Gold E. Activins and activin antagonists in the human ovary and ovarian cancer. Mol Cell Endocrinol. 2015;415:126-132.

33. Wildi S, Kleeff J, Maruyama H, Maurer CA, Büchler MW, Korc M. Overexpression of activin A in stage IV colorectal cancer. Gut. 2001;49(3):409-417.

34. Liu R, Wang JH, Xu C, Sun B, Kang SO. Activin pathway enhances colorectal cancer stem cell self-renew and tumor progression. Biochem Biophys Res Commun. 2016;479(4):715-720.

35. Kim H, Watkinson J, Varadan V, Anastassiou D. Multi-cancer computational analysis reveals invasion-associated variant of desmoplastic reaction involving INHBA, THBS2 and COL11A1. BMC Med Genomics. 2010;3:51.

36. Chen JL, et al. Differential effects of interleukin-6 and activin A in the development of cancer-associated cachexia. Cancer Res. 2016;76(18):5372-5382.

37. Togashi $\mathrm{Y}$, et al. Activin signal promotes cancer progression and is involved in cachexia in a subset of pancreatic cancer. Cancer Lett. 2015;356(2 pt B):819-827.

38. Loumaye A, et al. Role of activin A and myostatin in human cancer cachexia. J Clin Endocrinol Metab. 2015;100(5):2030-2038

39. Cho MS, et al. Autocrine effects of tumor-derived complement. Cell Rep. 2014;6(6):1085-1095.

40. Yeung TL, et al. TGF- $\beta$ modulates ovarian cancer invasion by upregulating CAF-derived versican in the tumor microenvironment. Cancer Res. 2013;73(16):5016-5028.

41. Cohen L, et al. Depressive symptoms and cortisol rhythmicity predict survival in patients with renal cell carcinoma: role of inflammatory signaling. PLoS One. 2012;7(8):e42324.

42. Radloff LS. The CES-D scale: A self report depression scale for research in the general population. Appl Psychol Meas. 1977;1:385-401.

43. Cutrona CE, Russell D. The provisions of social relationships and adaptation to stress. In: Jones, WH, Perlman D, eds. Advances in Personal Relationships. Greenwich, Connecticut, USA: JAI Press;1987:37-67.

44. Gyorffy B, Lánczky A, Szállási Z. Implementing an online tool for genome-wide validation of survival-associated biomarkers in ovarian-cancer using microarray data from 1287 patients. Endocr Relat Cancer. 2012;19(2):197-208. 